\title{
Optical coherence tomography angiography-based capillary velocimetry
}

\author{
Ruikang K. Wang \\ Qinqin Zhang \\ Yuandong $\mathrm{Li}$ \\ Shaozhen Song
}




\title{
Optical coherence tomography angiography-based capillary velocimetry
}

\author{
Ruikang K. Wang, ${ }^{\mathrm{a}, \mathrm{b}, \star}$ Qinqin Zhang, ${ }^{\mathrm{a}}$ Yuandong $\mathrm{Li}^{\mathrm{a}}{ }^{\text {and }}$ anhaozhen Song ${ }^{\mathrm{a}}$ \\ aUniversity of Washington, Department of Bioengineering, Seattle, Washington, United States \\ bUniversity of Washington, Department of Ophthalmology, Seattle, Washington, United States
}

\begin{abstract}
Challenge persists in the field of optical coherence tomography (OCT) when it is required to quantify capillary blood flow within tissue beds in vivo. We propose a useful approach to statistically estimate the mean capillary flow velocity using a model-based statistical method of eigendecomposition (ED) analysis of the complex OCT signals obtained with the OCT angiography (OCTA) scanning protocol. ED-based analysis is achieved by the covariance matrix of the ensemble complex OCT signals, upon which the eigenvalues and eigenvectors that represent the subsets of the signal makeup are calculated. From this analysis, the signals due to moving particles can be isolated by employing an adaptive regression filter to remove the eigencomponents that represent static tissue signals. The mean frequency (MF) of moving particles can be estimated by the first lag-one autocorrelation of the corresponding eigenvectors. Three important parameters are introduced, including the blood flow signal power representing the presence of blood flow (i.e., OCTA signals), the MF indicating the mean velocity of blood flow, and the frequency bandwidth describing the temporal flow heterogeneity within a scanned tissue volume. The proposed approach is tested using scattering phantoms, in which microfluidic channels are used to simulate the functional capillary vessels that are perfused with the scattering intralipid solution. The results indicate a linear relationship between the MF and mean flow velocity. In vivo animal experiments are also conducted by imaging mouse brain with distal middle cerebral artery ligation to test the capability of the method to image the changes in capillary flows in response to an ischemic insult, demonstrating the practical usefulness of the proposed method for providing important quantifiable information about capillary tissue beds in the investigations of neurological conditions in vivo. ๑ 2017 Society of Photo-Optical Instrumentation Engineers (SPIE) [DOI: 10.1117/1.JBO.22.6.066008]
\end{abstract}

Keywords: optical coherence tomography; optical coherence tomography angiography; functional monitoring and imaging; capillary velocimetry; eigendecomposition.

Paper 170109R received Feb. 15, 2017; accepted for publication May 19, 2017; published online Jun. 15, 2017.

\section{Introduction}

The development of optical coherence tomography $(\mathrm{OCT})^{1,2}$ has witnessed constant improvement in its imaging performances in terms of its resolution (up to $\sim 1 \mu \mathrm{m}$ ), ${ }^{3}$ sensitivity (up to $120 \mathrm{~dB}$ ), ${ }^{4-6}$ and imaging speed (up to $20 \mathrm{MHz}$ ). ${ }^{7-9}$ These promising developments clearly create new opportunities for researchers/scientists/physicians to investigate the dynamic blood flow responses at a micron scale, which otherwise would be difficult to assess using currently available biomedical imaging modalities. Previously, Doppler OCT (DOCT) or phase-resolved DOCT $^{10,11}$ has been developed to provide an ability to measure absolute flow velocity for application in retinal imaging, ${ }^{12-14}$ brain imaging, ${ }^{15}$ cochlear imaging, ${ }^{16}$ tissue engineering, ${ }^{17}$ etc. DOCT utilizes the phase difference (due to the Doppler effect) between adjacent A-lines acquired at one spatial location to estimate the axial velocity of moving particles. ${ }^{18,19}$ However, DOCT has limitations; for example, the estimation is highly dependent on the Doppler angle of the vessel of interest, which is difficult, if not impossible, to know in priori in practice. Another issue is its inability to provide either visualization or quantification of capillary blood flows because (1) the capillary flow is slow and (2) capillary beds are often approximately perpendicular to the incident OCT probe beam.

*Address all correspondence to: Ruikang K. Wang, E-mail: wangrk@u .washington.edu
OCT angiography (OCTA) is a recent addition to the OCT developments, for the purpose of visualizing functional blood flow within microcirculatory tissue beds in vivo, especially for imaging functional capillary vessels. ${ }^{20-29}$ Because it is fast, safe, noninvasive, and inexpensive, OCTA has now been used in biomedical imaging applications with promises for the investigations of tissue responses and injuries that have vascular involvements. The most exciting is its recent translation to clinical ophthalmology. ${ }^{30-34}$ There are a number of algorithms that have been introduced to generate the OCTA angiograms of perfused tissue beds, including methods that are based on OCT amplitude signal (e.g., speckle variance), ${ }^{24,25}$ phase signal (e.g., phase variance), ${ }^{26}$ and complex signal [i.e., combined use of the OCT amplitude and phase information, e.g., optical microangiography (OMAG)] ${ }^{21-23}$ All these algorithms are developed to detect the signal variations caused by moving red blood cells (RBCs), with the assumptions that the OCT signals that emerge from static tissues are static. ${ }^{21}$ Although promising and proven clinically useful in ophthalmology, none of these algorithms possess convincing ability to provide quantitative information about the capillary blood flow, which is, however, often required if one wants to understand how microcirculation works to maintain tissue integrity under both healthy and diseased conditions.

1083-3668/2017/\$25.00 @ 2017 SPIE 
The blood flow in the capillary beds is characterized by one-by-one RBC movement in the vessels, i.e., single-file flow. Furthermore, the single-file RBC flow is spatially and temporarily heterogeneous. ${ }^{35}$ There were several prior methods proposed in the development of OCT that attempt the quantification of RBC velocity and flux in single capillaries, for example, the methods that are based on the signal decorrelation time ${ }^{36-38}$ and amplitude peaks at capillary locations. ${ }^{39}$ While interesting, there remains a concern on whether these methods are valid in terms of the flow speeds within functional capillary vessels, which typically range from 0.3 to $5 \mathrm{~mm} / \mathrm{s} ;{ }^{40}$ furthermore, statistical evaluation of the capillary flows within scanned tissue beds remains cumbersome and time-consuming.

Multiple signal classification (MUSIC) based on eigendecomposition (ED) analysis is a method widely used in color Doppler ultrasound imaging. ${ }^{41}$ It is a super-resolution spectral estimation method based on the orthogonality of signal eigenvector subspaces. ${ }^{42}$ The MUSIC-based estimations of RBC flow flux within vessels have been applied to the OMAG dataset and validated by phantom studies and in vivo experiments in previous publications. ${ }^{42,43}$ However, using MUSIC to provide flow velocity is only amendable to relative large functional blood vessels, and there is no indication of its possibility to provide the capillary flow quantification within scanned tissue beds. In this paper, we propose an alternative model-based statistical analysis approach of ED-based analysis to estimate the mean frequency (MF) and frequency bandwidth (BF) of the dynamic OCT signals due to moving blood cells. The $\mathrm{MF}$ and BF indicate the mean velocity and the velocity heterogeneity of the blood flow within capillary tissue beds of the scanned tissue volume, respectively.

\section{Methods}

\subsection{Complex Optical Coherence Tomography Signal}

Assuming at time $t$ when the OCT system captures an A-scan signal, i.e., depth-scan, there are $M$ RBCs passing across this Ascan at various depths of $z_{i}$ and a speed of $v_{i}(i=0,1, \ldots, M)$. Without considering DC offsets, the spectral OCT signal of the A-scan at time $t$ can be expressed as

$$
\begin{aligned}
I(k, t)= & 2 S E_{R} \int_{-\infty}^{\infty} a(z) \cos (2 k n z) \mathrm{d} z \\
& +2 S E_{R} \sum_{0}^{M} b\left(z_{i}\right) \cos \left[2 k n\left(z_{i}-v_{i} t\right)\right]+\text { Noise }
\end{aligned}
$$

where $k$ is the wavenumber, $E_{R}$ is the reference light field, $S$ is the spectral density of the light source, $n$ is the refractive index of the tissue, and $z$ represents depth. $a(z)$ is the backscattering light field of tissue at the depth $z$. Here, we assume a uniform refractive index of the entire tissue, including blood, for simplicity. The first term is the contribution from the static components of the tissue sample. The second term represents the contribution of moving RBCs, i.e., blood flow signal. The detected signal also includes an additive noise due to system noise. It is not difficult to understand that if the A-scan is repeatedly captured at the same position, the first term in the left of Eq. (1) would be constant, while the second term varies with the time $t$. Therefore, the detected OCT signal consists of mixed signals between the static tissue signal and dynamic moving signal, as well as the system noise.

It is known from literature that the whole blood consists of only $\sim 3 \%$ to $7 \%$ in volume in the living body. The moving RBCs within blood act like scattering particles that give the dynamic OCT signal, which can be extracted to achieve OCTA. ${ }^{29}$ In the whole blood, the RBC concentration is about $45 \%$ in normal subjects, i.e., hematocrit. Therefore, from Eq. (1), it is not difficult to appreciate that the signal content within an OCT A-scan is dominated by the static tissue components. There are two models used to describe the backscattering of RBCs that contribute to OCT signal: (1) single backscattering model and (2) multiple backscattering model. A general agreement exists in both models that the scattering of light from the $\mathrm{RBCs}$ can be described by diffraction approximation, typically as a result of the convolution of the point spread function (PSF) with the blood cells. The PSF of probing light is a Gaussianshaped beam profile, indicating a Gaussian-shaped profile for the OCT signals appearing in the A-scan [or B-scan, threedimensional (3-D) scan], representing the backscattering of the moving RBCs within functional blood vessels. ${ }^{44}$

Temporarily, the OCTA signal captured from the moving RBCs overtime at one spatial location is a time-varying signal and depends on the direction and velocity of the RBCs passing through the OCT beam. Each scatterer (i.e., RBC) contributes to the total received time-varying OCTA signal, i.e., dynamic speckle signal, which is of an MF and a finite BF due to the limited observation time related to the movement through the sample volume. According to Brier, ${ }^{45}$ the dynamic speckle signal is equivalent to the laser Doppler. Therefore, the MF of the dynamic OCT signal would relate to the mean velocity of RBC movement within the tissue beds. The increase of flow heterogeneity within the microcirculatory tissue beds would increase the signal BF.

Static tissue is composed of a wide range of scatterers that have different scattering characteristics according to the Mie and Raleigh scattering theory. ${ }^{46,47}$ When light propagates within a homogeneous tissue volume containing a large amount of randomly distributed scatterers, a Gaussian-shaped signal results, which appear as speckles in the OCT images. Considering that the practical biological tissue is optically heterogeneous, the distribution of scatters is nonuniform within a larger tissue region, which would result in non-Gaussian distribution of the received tissue signal. However, when a smaller tissue region is considered, it would approach a uniform medium. In this case, the spatial distribution of received OCT signal from static tissue components would approximate a Gaussian distribution. An example is shown in Fig. 1, where the cerebral cortex of a mouse was imaged using a spectral domain OCT (SD-OCT) system. As expected, when the OCT signals within a smaller area of region 2 are analyzed, the distribution of the tissue signal indeed approaches a Gaussian shape; however, this distribution slightly deviates away from Gaussian for the relatively larger region (marked as region 1 ). Note that the analysis was performed on the OCT signals within the B-scan with the dynamic flow signal removed (using OMAG approach). ${ }^{28,29}$

Because the scatters within the tissue are of a wide range in size, they would contribute to a range of frequency components for the OCT signals due to their different characteristic behavior of either Raleigh or Mie scattering. In addition, the bulk tissue movement would contribute to the frequency components of the OCT signals. Compared to the dynamic signal from moving 

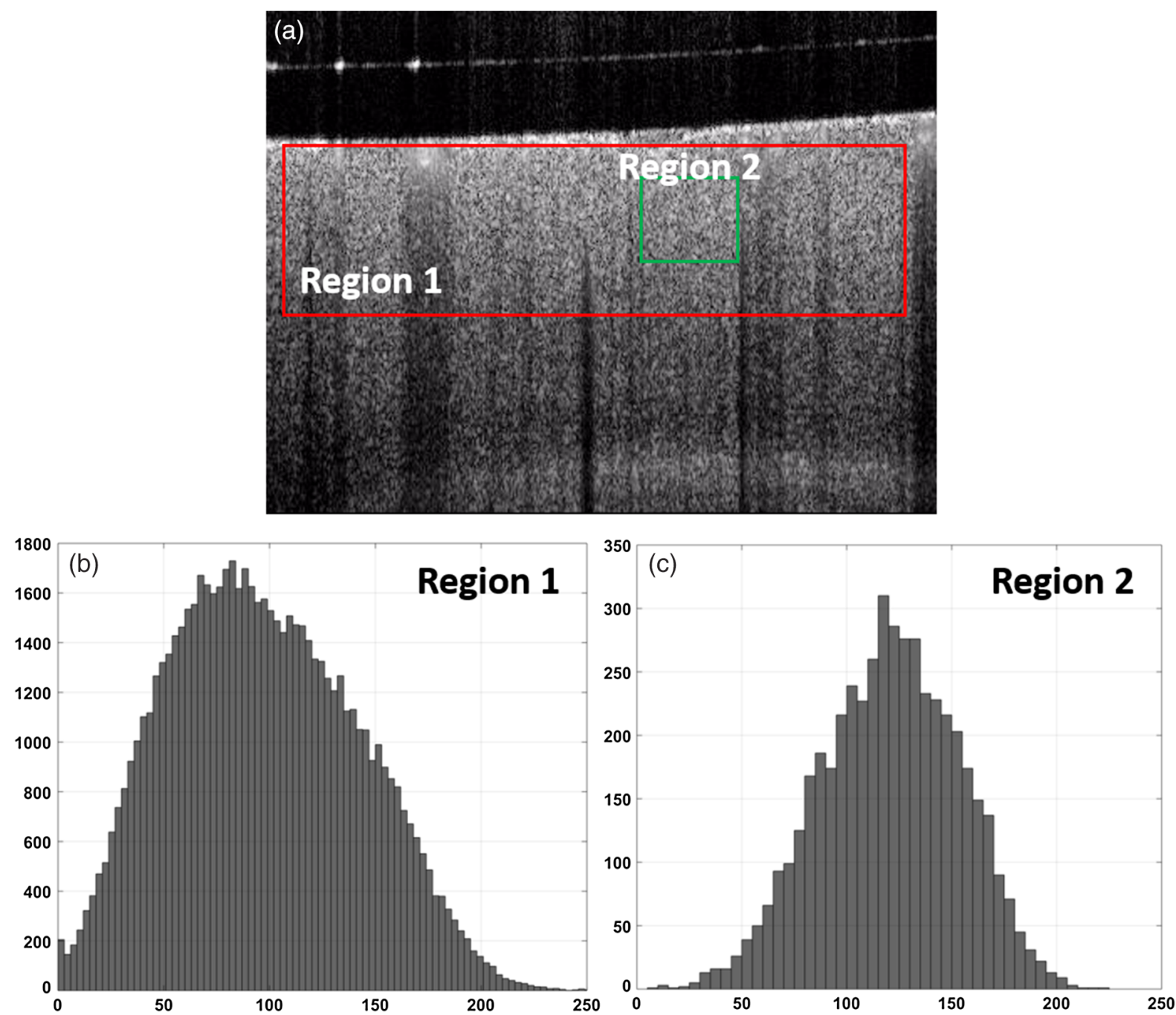

Fig. 1 The distribution of spatial tissue signals within OCT images captured from cerebral cortex in rodents. (a) Representative B-scan image. (b) and (c) The signal distribution within region 1 and region 2 marked in (a), respectively. The distribution of tissue signal in a smaller region approaches a Gaussian shape.

blood, the signal from static tissue would be of lower frequency and sets a lower bound for the flow signals.

\subsection{Signal Components and Decomposition}

In OCTA scanning protocol, to build up time-varying OCT signals from which the flow signal can be extracted, multiple Alines are acquired at the same spatial location. From the analysis above, this time-varying OCT signal, Eq. (1), consists of three components that represent the depth-resolved field speckle signals due to static tissue components, dynamic speckle signals due to moving blood cells, and additive system noise. We assume that statistically there exists no correlation between the speckle signal induced by blood flow and the speckle signal due to the background tissue. It would then be reasonable to consider that the dynamic blood flow signal superimposes on the tissue signal and that it disturbs the simplicity of the tissue signal. Therefore, the separation of tissue signal and blood signal by dimensional reduction can be achieved, assuming that the tissue signal arises from a low-dimensional space and the blood signal arises from a dynamic space with a much higher dimension. In this case, the problem can be reduced to the separation of a signal in a low-dimensional space from a more complex one. According to statistical signal processing, ${ }^{48}$ such problem solving can be well dealt with by eigendecomposition of the received signals.

Assuming there are $N$ repeated A-scans captured at one location, these complex OCT A-scan signals can then be collectively expressed as a two-dimensional vector form

$x=[x(1), x(2), \ldots, x(N)]^{\mathrm{T}}$,

where $N$ is the ensemble size with $x(i)$ representing the $i$ 'th A-scans $(i=1,2, \ldots, N)$. Being a sum of the signals from a large number of independent scatters, the central limit theorem applies, indicating that $x$ is a zero mean Gaussian random process. ${ }^{49}$ The vector $x$ has a complex Gaussian probability density function with zero mean given by

$f_{x}(x)=\frac{1}{\pi^{N}\left|R_{x}\right|} e^{-x^{* \mathrm{~T}} R_{x}^{-1} x}$

where $x^{* \mathrm{~T}}$ is the complex conjugated transpose (Hermitian transposition) of $x$ and the correlation matrix $R_{x}$ is defined as 
$R_{x}=E\left\{x x^{* T}\right\}$

where $E\{*\}$ is the expectation of the argument.

Because the OCT signal is the summation of the static component $\left(x_{s}\right)$, moving particle (RBCs) components $\left(x_{b}\right)$, and additive white noise $\left(x_{n}\right)$, the signal of each vector can be given as

$x=x_{s}+x_{b}+x_{n}$.

Assuming that these three signal components are statistically uncorrelated to each other, the correlation matrix of Eq. (5) can then be given by the following equation:

$R_{x}=R_{s}+R_{b}+\sigma_{\omega}^{2} I$,

where $R_{s}$ is the static correlation matrix, $R_{b}$ is the moving blood correlation matrix, $\sigma_{\omega}^{2}$ is the noise variance, and $I$ is the identity matrix.

To separate the OCT signals into its signal subsets, the discrete Karhunen-Loeve transform (DKLT) can be utilized; the DKLT orthogonally decomposes a general nonstationary random signal and can be considered a generalization of conventional Fourier analysis for nonstationary random processes. The DKLT is based on an eigenvector decomposition of the correlation matrix, where the eigenvectors and eigenvalues can be achieved by solving the equation

$R_{x} e_{i}=\lambda_{i} e_{i}$

The correlation matrix $R_{x}$ is the Hermitian symmetric and positive semidefinite. It is then possible to find $N$ orthonormal eigenvectors $e_{1}, e_{2}, \ldots, e_{N}$ and corresponding eigenvalues $\lambda_{1}, \lambda_{2}, \ldots, \lambda_{N}$, which are real and nonnegative. The correlation matrix can be written with eigenvalues and eigenvectors in the following form:

$R_{x}=E \Lambda E^{* T}$,

where $\Lambda$ and $E$ are the eigenvalue $\left(\lambda_{1}>\lambda_{2}>\cdots>\lambda_{N}\right)$ and eigenvector matrices, respectively, defined as

$$
\Lambda=\left[\begin{array}{cccc}
\lambda_{1} & 0 & \ldots & 0 \\
0 & \lambda_{2} & \ldots & 0 \\
\vdots & \vdots & & \vdots \\
0 & 0 & \ldots & \lambda_{N}
\end{array}\right], \quad E=\left[\begin{array}{cccc}
\mid & \mid & & \mid \\
e_{1} & e_{2} & \ldots & e_{N} \\
\mid & \mid & & \mid
\end{array}\right]
$$

The total energy in the received OCT signal equals the sum of all the eigenvalues. Thus, the eigenvalue distribution must be a measure of the signal spectrum. In this case, the eigenvalue spectrum is a generalization of the Fourier power spectrum. An example of eigenvalue spectrum from the three components is shown in Fig. 2 for a typical cortical tissue in rodents, where the largest energy is dominated by the static signal located at the first eigenvalue. However, the moving blood signals are distributed in the subsequent eigenvalues with relative energy higher than that of static tissue components. The noise components are almost equally distributed across the spectrum, which is a typical behavior of the system noise.

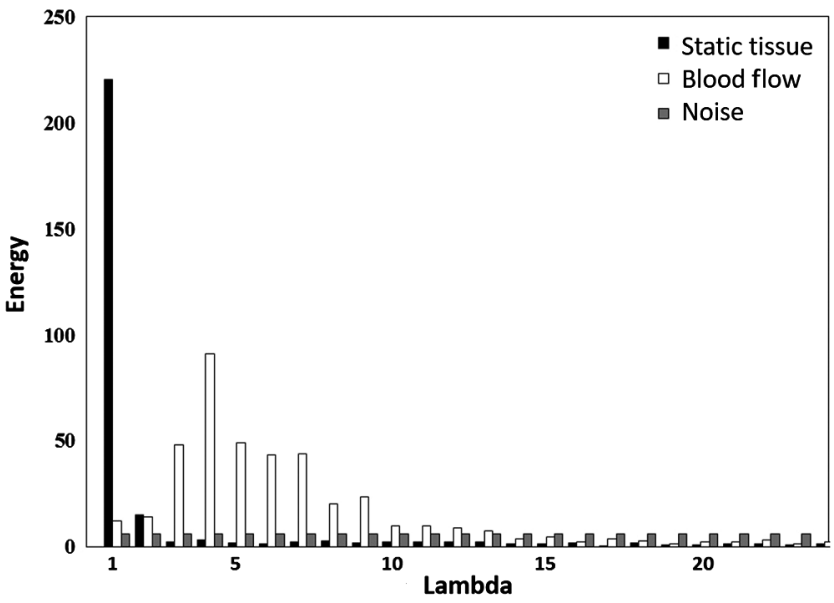

Fig. 2 Typical eigenvalue spectrum, where 30 eigenvalues are analyzed for the signals representing static tissue components, dynamic motion signal, and noise. The OCT signals are captured from the cortical tissue of a rodent.

As discussed above, the DKLT expansion is to decompose the signal into the orthogonal eigenvectors. Each eigenvector and its corresponding eigenvalue represent a subset of the signal to be analyzed. The highest eigenvalue contains the highest energy content of the signal. The eigenvector of the highest eigenvalue describes the spectral content of the signal with the highest energy. The frequency transform of the eigenvector gives the spectrum of the corresponding signal component. Therefore, each eigenvector is related to a specific frequency band. From the eigenvalue/energy spectrum, the static signal originates from stationary and slowly moving tissue or particles, which dominate in the low frequency and concentrate in a few eigenvalues corresponding to low-frequency eigenvectors. By contrast, the signal from the faster moving blood has most of the energy concentrated along eigenvectors with higher frequency component. The noise has equal energy along all of the eigenvectors. This is evidenced from Fig. 2 for brain imaging of cortical tissue in rodents. Therefore, the static component can be maximally suppressed to retain the flow signals with minimal distortion through applying the eigenregression filtering, ${ }^{50}$ where the filter identifies and removes eigencomponents that represent static tissue signal. Assuming that $K c$ is eigencomponents corresponding to static tissue signal, the eigenregression filter can be interpreted as having a filter order of $(K c-1)$. From Fig. 2, for rodent brain imaging, $K c=1$ would be sufficient to remove the static signals from the OCT signals to contrast the blood flow. This static tissue signal suppression approach is inherently adaptive to the repeated A-scans (or repeated B-scans) because its attenuation response of the regression filter is defined according to the eigencomponents in the ensemble signal composition.

The power spectrum of the signal is the Fourier transform of the autocorrelation function defined as

$G_{x}(\omega)=\int_{-\infty}^{\infty} R_{x}(\tau) e^{-i w \tau} \mathrm{d} \tau$.

If the frequency components have spectral support on the interval $(-\pi, \pi)$, the signal power indicating the presence of blood flow, i.e., OCTA signal, can be simplified as follows: 


$$
P=\int_{-\pi}^{\pi} G(\omega) \mathrm{d} \omega
$$

The MF of signal frequency spectrum, $\omega_{m}$, representing the mean velocity of blood flow within scanned tissue volume and the $\mathrm{BF}, B^{2}$, of the frequency spectrum, which is related to the flow fluctuation (temporal heterogeneous of flow) can be defined as follows:

$$
\begin{aligned}
& \omega_{m}=\frac{1}{P} \int_{-\pi}^{\pi} \omega G(\omega) \mathrm{d} \omega \quad \text { and } \\
& B^{2}=\frac{1}{P} \int_{-\pi}^{\pi}\left(\omega-\omega_{d}\right)^{2} G(\omega) \mathrm{d} \omega .
\end{aligned}
$$

The estimation of spectral moments can be obtained using the well-known lag-one autocorrelation equation ${ }^{51}$ given by the following equation:

$$
\begin{aligned}
& \omega_{k}=\frac{F P S / 2}{2 \pi} \arg \left\{R(1)_{k}\right\}, \\
& \text { for } R(1)_{k}=\frac{1}{N_{D}-1} \sum_{m=0}^{N_{D}-2} e_{k}^{*}(m) e_{k}(m+1),
\end{aligned}
$$

where $R(1)_{k}$ is the lag-one autocorrelation value for the $k$ 'th eigenvector, FPS is the sampling rate, and $N_{D}$ is the number of eigenvectors. We investigate the frequency content of the individual eigenvectors, upon which the spectral band information as well as the eigencomponents that represent them are obtained. One frequency measure of interest is each eigenvector's MF. Figure 3 provides the relationship between frequency and eigenvectors, presented in box-whisker plot form, ${ }^{52}$ which

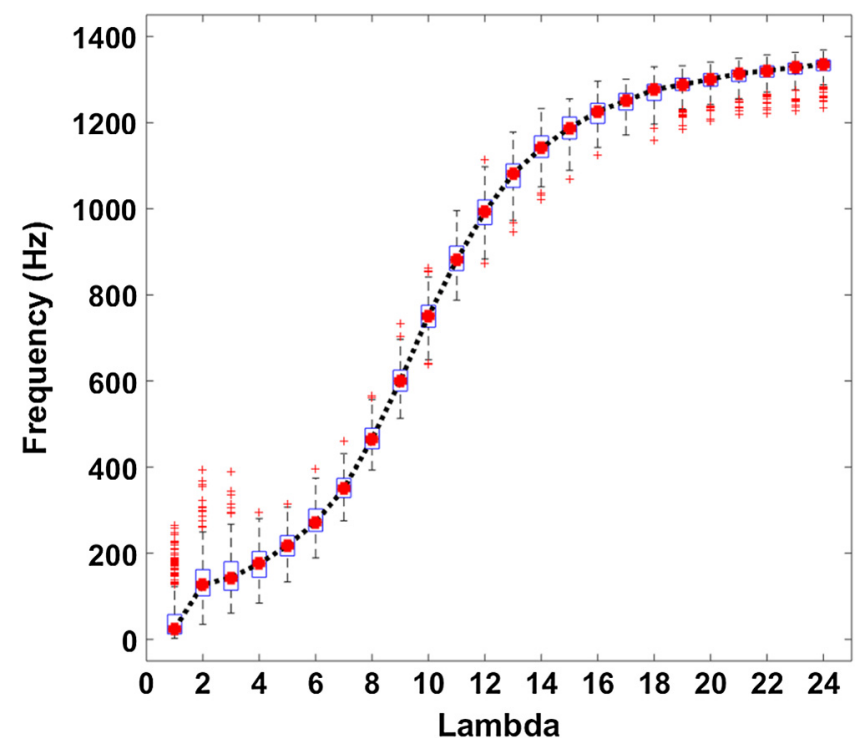

Fig. 3 The relationship between frequency and eigenvalue (eigenvector) presented in box-whisker plot form. The results were obtained and evaluated from 10 3-D scans of rodent brain in vivo. were evaluated from 10 3-D scans from rodent brain in vivo. The red squares connected with a black dash line indicate the MF of each eigenvector. Combined with the energy spectrum of Fig. 2, a conclusion can be made that the highest energy is associated with the highest eigenvalue, representing the static or slowing moving signal, corresponding to the eigenvector of lowest frequency $(K c=1)$. Additionally, the signals from the moving blood were mainly concentrated in higher frequencies $(K c>1)$.

\section{Experimental System Setup and Design}

\subsection{System Setup Used in the Study}

For all the results reported in this study, we used a fiber-based SD-OCT system that was previously described in Ref. 53. Briefly, the system employed a superluminescent diode (Thorlabs Inc., Newton, New Jersey) that has a central wavelength of $1340 \mathrm{~nm}$ with a bandwidth of $110 \mathrm{~nm}$, providing $\mathrm{a} \sim 7-\mu \mathrm{m}$ axial resolution in the air. In the sample arm, a $10 \mathrm{x}$ scan objective lens was used to deliver the light onto the sample, achieving a lateral resolution of $7 \mu \mathrm{m}$ (full width at half maximum). The detection system was a fast spectrometer that employed a line scan camera (Goodrich Inc., Princeton, New Jersey) with a line scan rate of $92 \mathrm{kHz}$ to capture the spectral interferograms formed between the reference light and the sample light. With a probe light power of $3.5 \mathrm{~mW}$ at the sample surface, the system had a measured dynamic range of $105 \mathrm{~dB}$. The operations for probe beam scanning, data acquisition, and data storage were controlled by a custom software package written in Labview.

\subsection{Scanning Protocol}

To quantify the capillary blood flow faithfully, the system is required to capture OCT signals that represent dynamic speckle signals due to the blood flow without losing its information content. Capillary flow is reported to possess a wide velocity range, from hundreds of micrometers per second to a few millimeters per second..$^{40}$ According to the study conducted by Choi et al., ${ }^{53}$ the time interval of $50 \mu \mathrm{s}$ to contrast blood flow for OCTA is required to establish a quantitative relationship between dynamic OCT signals and vascular flow up to $5 \mathrm{~mm} / \mathrm{s}$. To fulfill this requirement, we employed an M-B scan protocol on the SDOCT system with a time interval ranging from $50 \mu$ s to $2.5 \mathrm{~ms}$ to detect both fast (up to $5 \mathrm{~mm} / \mathrm{s})$ and slow flow $(\sim 100 \mu \mathrm{m} / \mathrm{s})$, simultaneously. According to these parameters, 50 A-scans were required to repeat at the same spatial position with an A-scan rate of $20 \mathrm{kHz}$. Two hundred A-scans formed one $\mathrm{B}$-scan in the fast axis ( $x$-direction) with an imaging region of $1.4 \mathrm{~mm}$. In the slow axis ( $y$-direction), 200 sampling B-scans were captured to cover $1.4 \mathrm{~mm}$.

After 3-D volumetric data acquisitions using the scanning protocol above, the following analyses were conducted to generate the vascular image (dynamic signal power), the MF (related to capillary velocity), and BF of blood flow (related to the temporal bandwidth of capillary flows). Note that, in the analysis of animal results below, the relative large vessels ( $>30 \mu \mathrm{m}$ ) were excluded from the analysis because the flows in these vessels are likely fast, which would probably exceed the flow measurement limit using the current scanning protocol (i.e., $5 \mathrm{~mm} / \mathrm{s}$ ). 


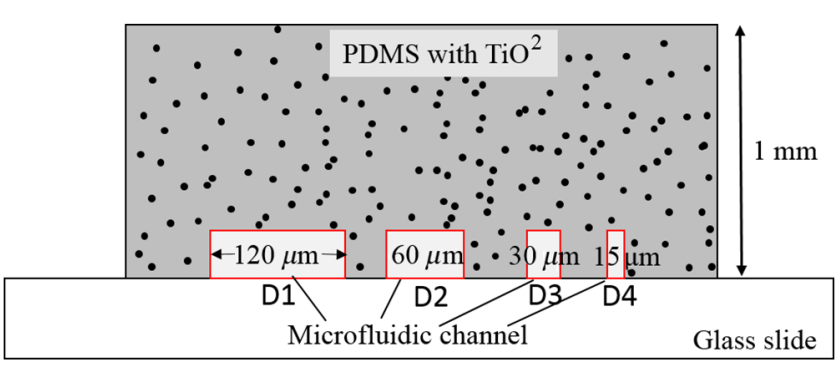

Fig. 4 Cross-sectional view of the microfluidic phantom. Four microfluidic channels with the sizes as shown were fabricated at the interface between the channel body and the substrate of glass slide. The channel body was fabricated with polydimethylsiloxane (PDMS) mixed with $\mathrm{TiO}_{2}$ powder to mimic static tissue surrounding blood vessels.

\subsection{Phantom Study}

To validate the proposed ED-based quantification approach, we first conducted phantom experiments using microfluidic channels perfused with $5 \%$ intralipid solutions with predefined flow velocity in each microfluidic channel. The details of the microfluidic flow phantom and its fabrication were described in the previous study. ${ }^{53}$ Briefly, the scattering phantom consists of four equal-height $(40 \mu \mathrm{m})$ microfluidic channels at its interface with a glass substrate, shown in Fig. 4. The sizes of the channels are $120 \mu \mathrm{m}$ (D1), $60 \mu \mathrm{m}$ (D2), $30 \mu \mathrm{m}$ (D3), and $15 \mu \mathrm{m}$ (D4) in width, respectively. The imaging protocol above covered all the channels in each imaging scan. The flow of $5 \%$ intralipid in the microfluidic channel was driven by an external precision syringe pump. Since the channel design was based on a dimidiate and symmetric strategy, the flow parameters in each subchannel are predictable. Moreover, the symmetric design provides an important feature in the flow-field distribution, for which the average flow velocity is constant throughout the microfluidic channels with different sizes. Table 1 lists the predictable flow rate and

Table 1 Parameters of pump rate and corresponding flow rate and average velocity in the four channels.

\begin{tabular}{|c|c|c|c|c|c|c|c|c|}
\hline \multirow{2}{*}{$\begin{array}{l}\text { Pump rate } \\
(\mu \mathrm{L} / \mathrm{h})\end{array}$} & \multicolumn{4}{|c|}{ Flow rate $(\mu \mathrm{L} / \mathrm{h})$} & \multicolumn{4}{|c|}{$\begin{array}{l}\text { Average velocity } \\
\qquad(\mathrm{mm} / \mathrm{s})\end{array}$} \\
\hline & D1 & D2 & D3 & D4 & D1 & D2 & D3 & D4 \\
\hline 3.46 & 1.76 & 0.88 & 0.44 & 0.22 & 0.1 & 0.1 & 0.1 & 0.1 \\
\hline 10.37 & 5.20 & 2.60 & 1.30 & 0.65 & 0.3 & 0.3 & 0.3 & 0.3 \\
\hline 17.28 & 8.64 & 4.32 & 2.16 & 1.08 & 0.5 & 0.5 & 0.5 & 0.5 \\
\hline 27.65 & 13.84 & 6.92 & 3.46 & 1.73 & 0.8 & 0.8 & 0.8 & 0.8 \\
\hline 41.47 & 20.72 & 10.36 & 5.18 & 2.59 & 1.2 & 1.2 & 1.2 & 1.2 \\
\hline 51.84 & 25.92 & 12.96 & 6.48 & 3.24 & 1.5 & 1.5 & 1.5 & 1.5 \\
\hline 62.21 & 31.12 & 15.56 & 7.78 & 3.89 & 1.8 & 1.8 & 1.8 & 1.8 \\
\hline 69.12 & 34.56 & 17.28 & 8.64 & 4.32 & 2.0 & 2.0 & 2.0 & 2.0 \\
\hline 103.68 & 51.84 & 25.92 & 12.96 & 6.48 & 3.0 & 3.0 & 3.0 & 3.0 \\
\hline 138.24 & 69.12 & 34.56 & 17.28 & 8.64 & 4.0 & 4.0 & 4.0 & 4.0 \\
\hline 172.8 & 86.40 & 43.20 & 21.60 & 10.80 & 5.0 & 5.0 & 5.0 & 5.0 \\
\hline
\end{tabular}

average velocity in each of the four channels at a range of pump rates. These pump rates covered a flow velocity range from 0.1 to $5 \mathrm{~mm} / \mathrm{s}$, which were used in experimental verification to establish the mean velocity measured in each channel through the methods described above.

\subsection{Distal Middle Cerebral Artery Occlusion}

For the observation of cerebral capillary flow response to ischemic stroke, dMCAO was used as a rodent stroke model in which permanent focal ischemia in the cerebral cortex is induced through ligating the distal middle cerebral artery (dMCA). ${ }^{54}$ C57BL/6 mice (23 to $25 \mathrm{~g}$ ) were prepared under isoflurane anesthesia $(1.5 \%$ to $2.0 \%$ in $0.2 \mathrm{~L} / \mathrm{min}$ oxygen, $0.8 \mathrm{~L} / \mathrm{min}$ air) and placed on a stereotaxic frame. The body temperature of the mouse was maintained at $36.8^{\circ} \mathrm{C}$ by a heating blanket on the frame whose temperature was controlled by a homeothermic monitoring system (50-7220F, Harvard Apparatus) that continually monitored the body temperature using rectal insertion of a temperature-sensing probe. Under anesthesia, the mice received an open skull craniotomy, ${ }^{55,56}$ where a $4 \mathrm{~mm} \times 4 \mathrm{~mm}$ area of the skull over the somatosensory cortex in right hemisphere was removed along with the dura and then the exposed somatosensory cortex was covered with a 5-mm-diameter transparent glass coverslip. The cranial window covered the territories supplied by the anterior cerebral artery and middle cerebral artery, as well as anastomoses. Following the surgery, OCTA imaging of the cortex according to the scanning protocol described in Sec. 3.2 was performed to obtain the baseline scan. Then, dMCAO was induced, in which a $1-\mathrm{cm}$ skin incision was made between the right ear and eye of the mouse and the temporal muscle below the skin was removed. Then, the temporal bone overlying the dMCA was slightly thinned to expose the dMCA. The exposed artery was permanently occluded using a microbipolar electrocoagulator (Aaron 940 ${ }^{\mathrm{TM}}$, Bovie Medical Corp.). Following this procedure, the animal was OCTA-scanned again to obtain the capillary flow responses to the dMCAO ischemic injury. All experimental procedures performed in this study were approved by the Institutional Animal Care and Use Committee of the University of Washington (Protocol No. 4262-01).

\section{Results}

\subsection{Phantom Verification}

The quantification algorithm was applied on the 3-D data acquired from the microfluidic phantom to obtain the OCT structural image [Fig. 5(a)], static tissue signal [Fig. 5(b)], and the flow signal power [Fig. 5(c)], which contributes to OCTA flow signal. According to the analysis demonstrated in Fig. 3, each eigenvector corresponds to a specific frequency or velocity in each single channel. By averaging all the frequencies in each channel, the MF can be obtained for each pump rate applied to the microfluidic channels. After calculating the signal power and MF with different pump rates, the relationship of velocity with signal power/intensity and MF was obtained and summarized in Figs. 5(d) and 5(e). In Fig. 5(d), the signal power decomposed by the ED-algorithm is related to the OCTA signal magnitude/intensity that is seen to increase with the increase of flow velocity and asymptotically approaches a plateau, which agreed well with the results reported in previous publications ${ }^{38,53}$ indicating its relationship with flow flux. In Fig. 5(e), 

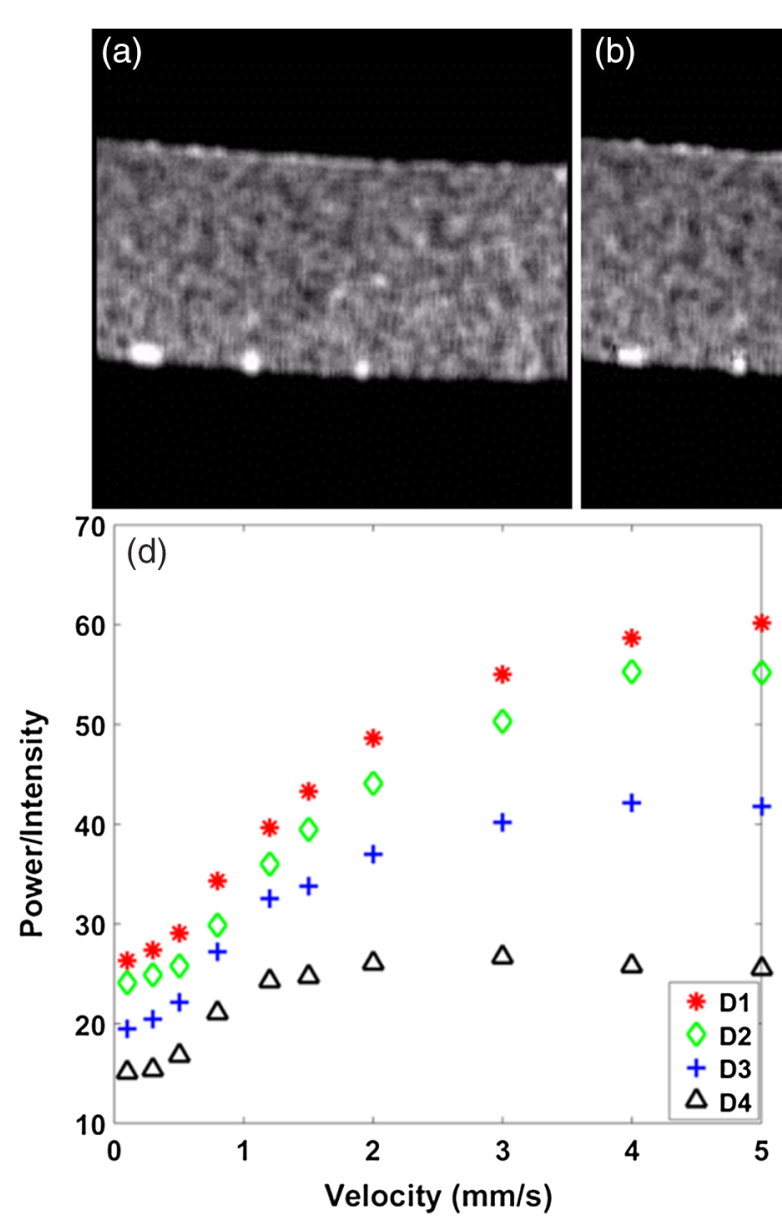
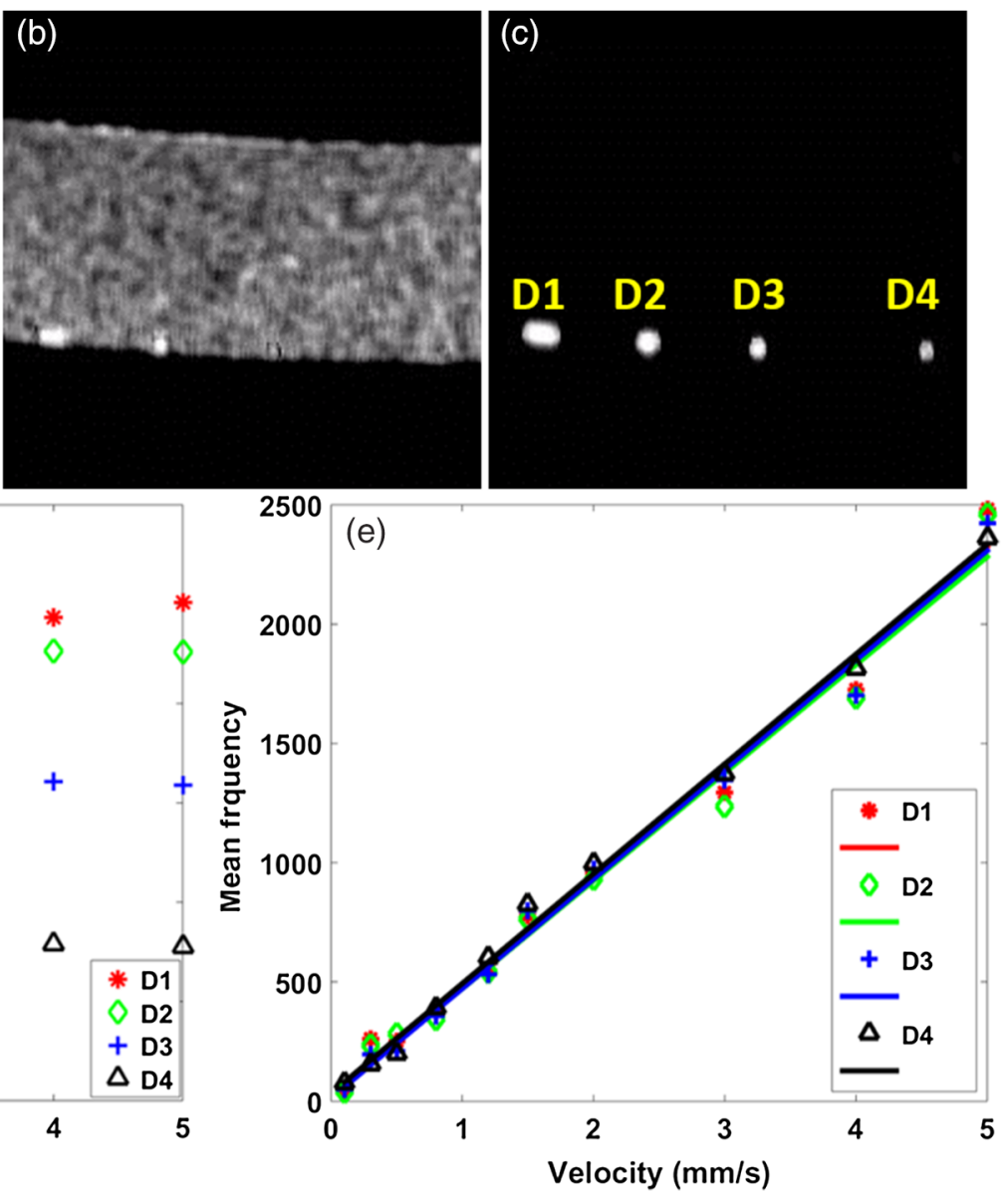

Fig. 5 The ED-algorithm is capable of measuring the mean flow velocity in the microfluidic channels. (a) The structure OCT image, (b) static tissue signal image, and (c) OCTA flow image of the scanned microfluidic phantom. (d) The relationship between velocity and OCTA signal power within four channels. (e) The relationship between velocity and MF for four channels. A same linear relationship between MF and velocity is found with squared Pearson's correlation coefficient of 0.983 of D1 channel, 0.982 of D2 channel, 0.970 of D3 channel, and 0.920 of D4 channel among all the given velocities.

the relationship between MF and flow velocity among all the channels is shown to possess the same linear relationship with squared Pearson's correlation coefficient of 0.983 of D1 channel, 0.982 of D2 channel, 0.970 of D3 channel, and 0.920 of D4 channel among all the velocities given. Although the exact relationship between MF and the flow velocity remains to be explored, the one-to-one relationship of MF to the flow velocity regardless of the channel sizes demonstrates that the ED-quantification algorithm is able to measure accurately the velocities in capillaries with different sizes.

\subsection{Capillary Flow Responses to Distal Middle Cerebral Artery Occlusion Ischemic injury}

After demonstration on microfluidic phantom to verify the linear relationship of measured average frequencies of dynamic OCT signals with the predefined flow velocity in the microfluidic channels, we investigate whether the proposed ED-quantification algorithm is able to detect the cerebral capillary response to ischemic injury (dMCAO model). The capillary flows within cortical tissue beds would exhibit heterogeneity. Ischemic injury would modify this heterogeneous property of the cerebral capillary flow. In the experiment, we selected two regions in the affected brain region to provide quantitative assessments of the changes in cerebral vessel morphology and capillary flow response to the dMCAO. The purpose of this choice was to check the consistency of quantification because the responses in these two regions must be approximately the same.

The changes in cerebral tissue and blood vessel morphology before and after the dMCAO are summarized in Fig. 6. The scanning positions at two selected locations were shifted. This was due to the animal being required to be removed from the imaging platform to the surgery table for the surgical ligation of the $\mathrm{dMCA}$ to induce $\mathrm{AMCAO}$, and then placed back on the imaging platform for the next imaging session. We tried our best to colocate the regions for baseline scan and dMCAO scan, but a shift in position was eminent due to lack of an automated tracking mechanism in our system setup. Despite these, the decrease of the blood flow and capillary density was clearly observed on OCTA images when comparing the results before [Figs. 6(a)-6(d)] and after the dMCA ligation [Figs. 6(e)-6(h)].

Quantitative analysis was performed to calculate the changes in vessel density before and after the dMCA ligation. The morphological parameter of vessel density was defined as the ratio of the area occupied by blood vessels to the total scanned area. ${ }^{57,58}$ Due to the shift of the scanned position, proper quantification required coregistration so that the comparison was made for the same region [i.e., region of interest (ROI)] before 
Location 1
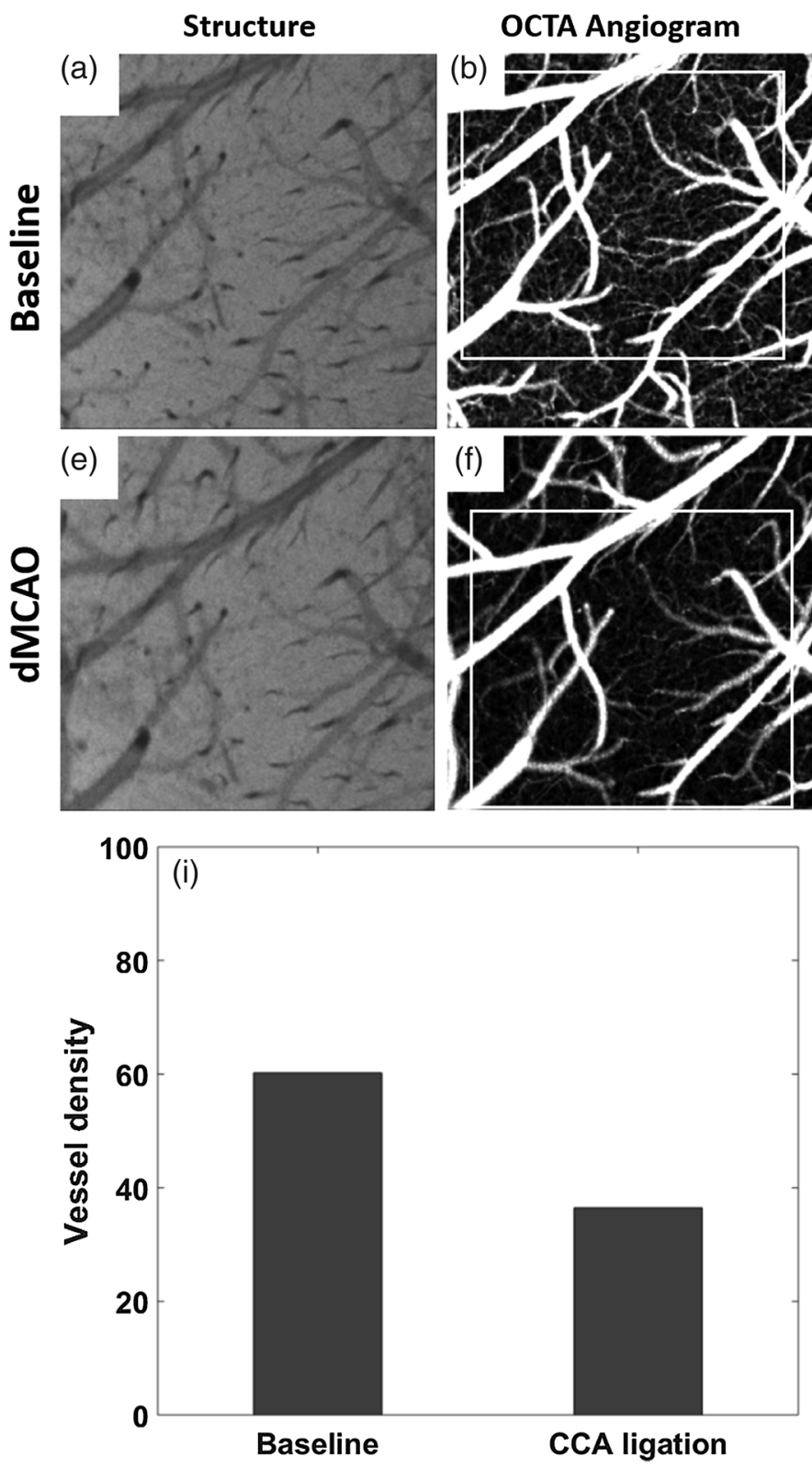

OCTA Angiogram
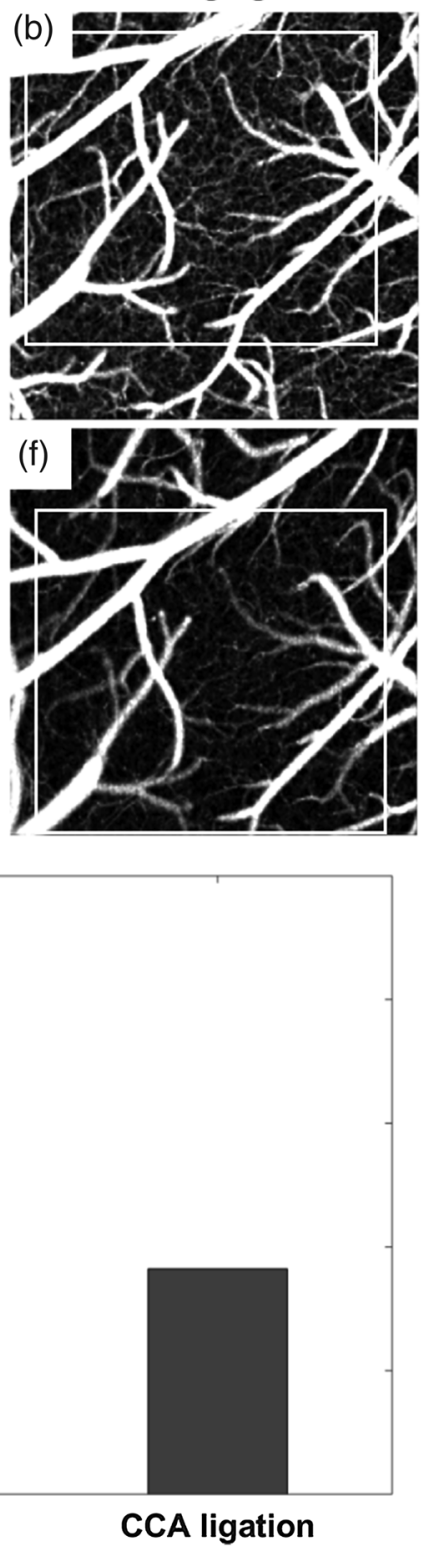
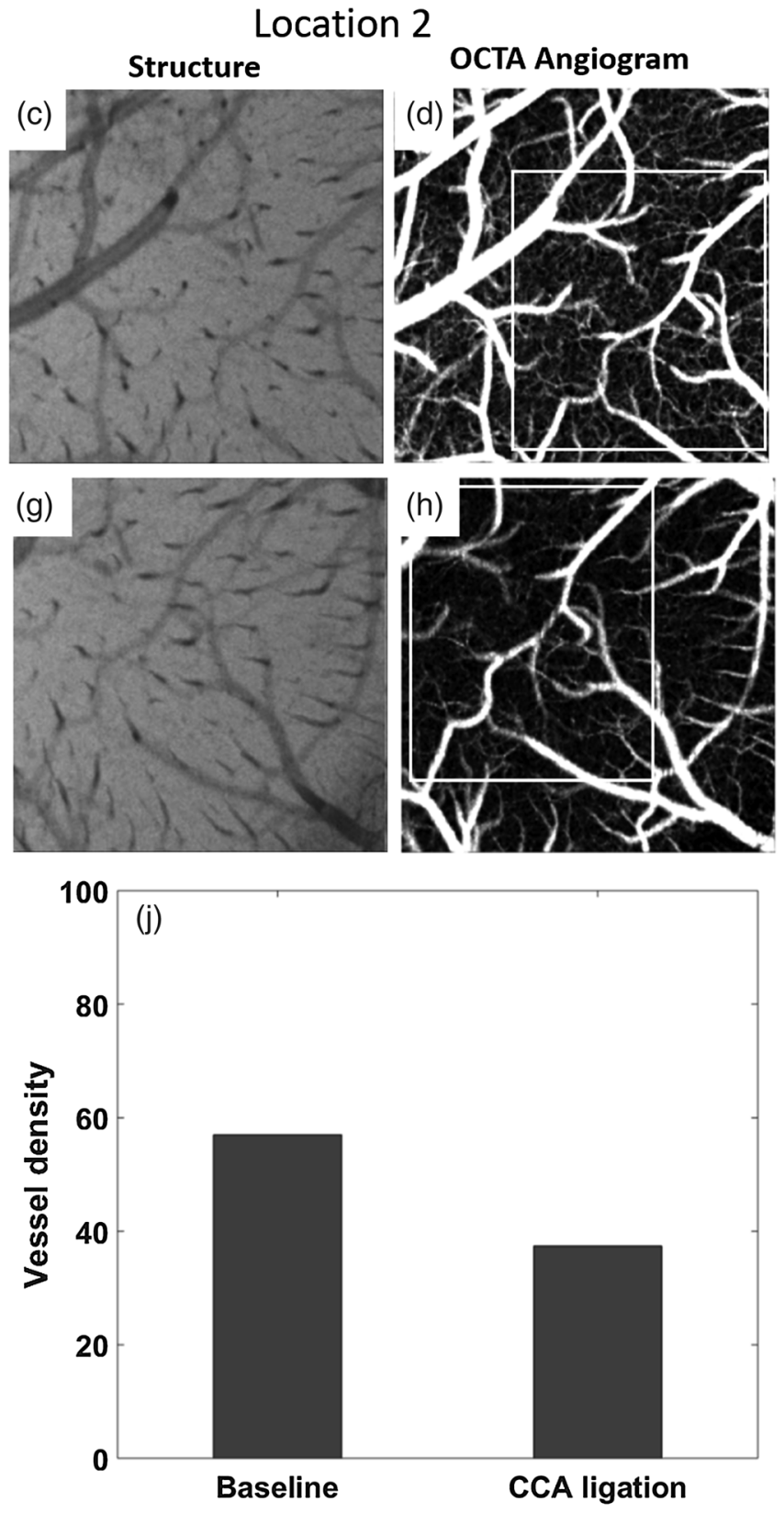

Fig. 6 The ED-based algorithm is capable of visualizing and quantifying the changes in capillary vessel density after the dMCA ligation in the mouse brain. The results are presented for two selected locations (left: location 1 and right: location 2) within the dMACO affected brain regions before (top row) and after (middle row) the dMCA ligation. (a, c, e, g) The reflectance and (b, $d, f, h)$ OCTA enface images are given side-by-side. For quantification of vessel density, the ROls (marked as white boxes) are selected through coregistration of OCTA angiograms obtained at location $1(b, e)$ and location $2(d, h)$ for the comparison between baseline and dMCAO. The quantification results are shown in (i) for location 1 and (j) for location 2. A reduction of vessel density about 30 percent was found for both ROls after the dMCA ligation. The enface image size is $1.4 \times 1.4 \mathrm{~mm}^{2}$.

and after the dMCA ligation. This was done automatically by an in-house software. The ROI coregistration is shown as the white boxes in the pair of images in Fig. 6, upon which the vessel density was evaluated. The results are shown in Figs. 6(i) and 6(j) for location 1 and location 2, respectively, both giving a $\sim 30$ percent reduction in vessel density after the dMCA ligation, demonstrating that the dMCA ligation in the current experiment caused the cessation of $\sim 1 / 3$ rd capillary blood flow in the cortical tissue, giving rise to mild ischemia in the affected regions.
The MF map and BF map were demonstrated in Fig. 7 before and after the dMCA ligation for both the locations. In Fig. 7, the cross-sectional images at the position marked with the white lines in the MF maps are also given, where structural (typical OCT cross-section image), blood flow (OCTA cross section), $\mathrm{MF}$, and $\mathrm{BF}$ images are provided side-by-side for detailed scrutiny. The OCT angiograms shown in Fig. 6 do not provide direct information about capillary velocity or flow information before and after ligation. With the ED-based quantification algorithm, the reduction of capillary flows was observed on enface MF 

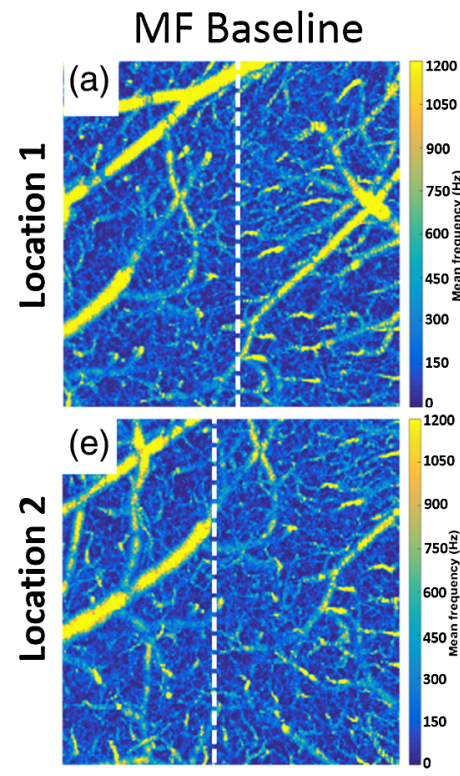

MF dMCAO

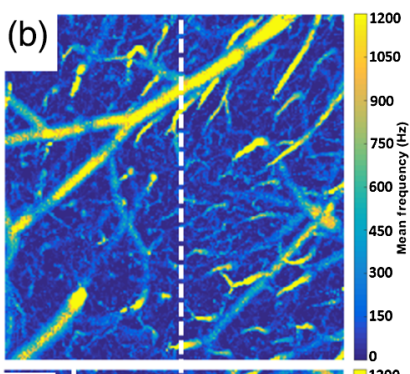

1050 (f)

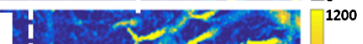

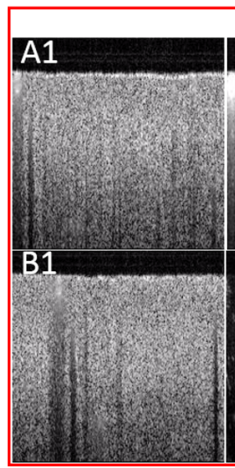

Location 1
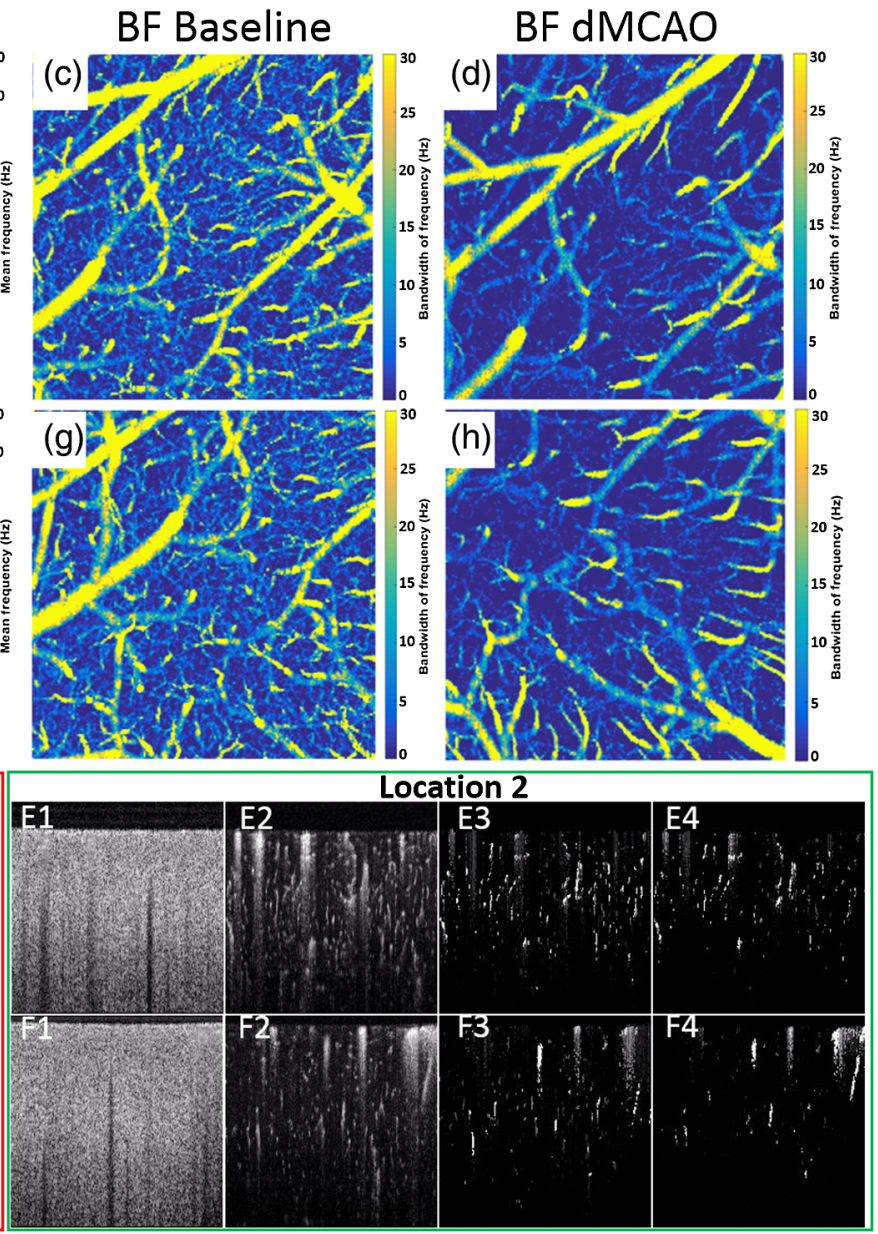

Fig. 7 The ED-based algorithm is capable of visualizing and quantifying the changes in capillary flows after the dMCA ligation in the mouse brain. (a, b, e, and f) The MF maps and (c, d, g and h) BF maps are given for both locations 1 and 2 . The cross-sectional images of structural, blood flow, MF, and its bandwidth at the positions marked as dashed line in (a, b, e, and f) are given at the bottom row for locations 1 and 2, respectively. Location 1 (red box): before ligation, (a1 to a4); after ligation: (b1 to b4). Location 2 (green box): before ligation, (e1 to e4); after ligation: (f1 to f4). The enface image size is $1.4 \times 1.4 \mathrm{~mm}^{2}$.

maps through comparing the results before and after ligation [Figs. 7(a) and 7(b), Figs. 7(e) and 7(f)]. According to the microfluidic phantom study, the current scanning protocol with a $20-\mathrm{kHz}$ A-line rate is able to provide the measurement of the velocity range from 0 to $5 \mathrm{~mm} / \mathrm{s}$, in which range the velocity is linear with the mean frequencies (from 0 to $\sim 2000 \mathrm{~Hz}$ ). The MF range on the mouse brain shown in Fig. 7 is observed from 0 up to $\sim 1200 \mathrm{~Hz}$, which corresponds to the velocity in a range from 0 to $\sim 3 \mathrm{~mm} / \mathrm{s}$. BF maps reflect the temporal heterogeneity of capillary flows. This heterogeneous property is clearly observed in capillaries or penetrating capillaries, especially in the downstream of capillaries after dMCA ligation [see Figs. 7(c), 7(d), 7(g), and 7(h)]. Comparing the results before and after the dMCA ligation, the velocities of capillary flows were decreased with the induction of dMCAO in the brain region, as was the temporal heterogeneity of the capillary flows. Note that the vessels with a size more than $30 \mu \mathrm{m}$ were excluded in the assessments, where the blood flow likely exceeded the measurement limit of the current scanning protocol.

To investigate how the capillary flow responds to the ischemic injury, we attempted to statistically quantify the velocity changes among the capillaries within the scanned tissue beds. In doing so, the flow histograms of both MF maps and BF maps in the same ROIs before and after dMCA ligation for both location 1 and location 2 were calculated and displayed in Fig. 8, respectively, indicating the velocity distribution for all the capillaries investigated. Note that the assessment excluded the vessels with a size more than $30 \mu \mathrm{m}$. Histogram distribution gives a nice way to show the spatial heterogeneity property of the capillary flow [Figs. 8(a) and 8(c)]. After the dMCA ligation, the reduction of capillary flows is clearly seen, but the capillary flows still possess the property of spatial heterogeneity. To show more clearly how the capillary flow is reduced, we performed differentiation of the histogram functions between before and after the dMCA ligation. The results are provided as insets in Fig. 8, where we can conclude that after the dMCA ligation, the capillary flows in most of the capillaries are statistically moving to slow velocities, while the counts of faster flow velocity become less. The same is true for the temporal heterogeneity of the capillary flows [Figs. 8(b) and 8(d)]. Such behavior of the capillary blood flow is characteristic for the ischemic injury because of the lack of perfusion from the dMCA. We understand that the capillary flow responses and its characteristic heterogeneity property are extremely complicated and that may be related to how the brain tissue is trying to utilize and extract the oxygen from the blood to support the 

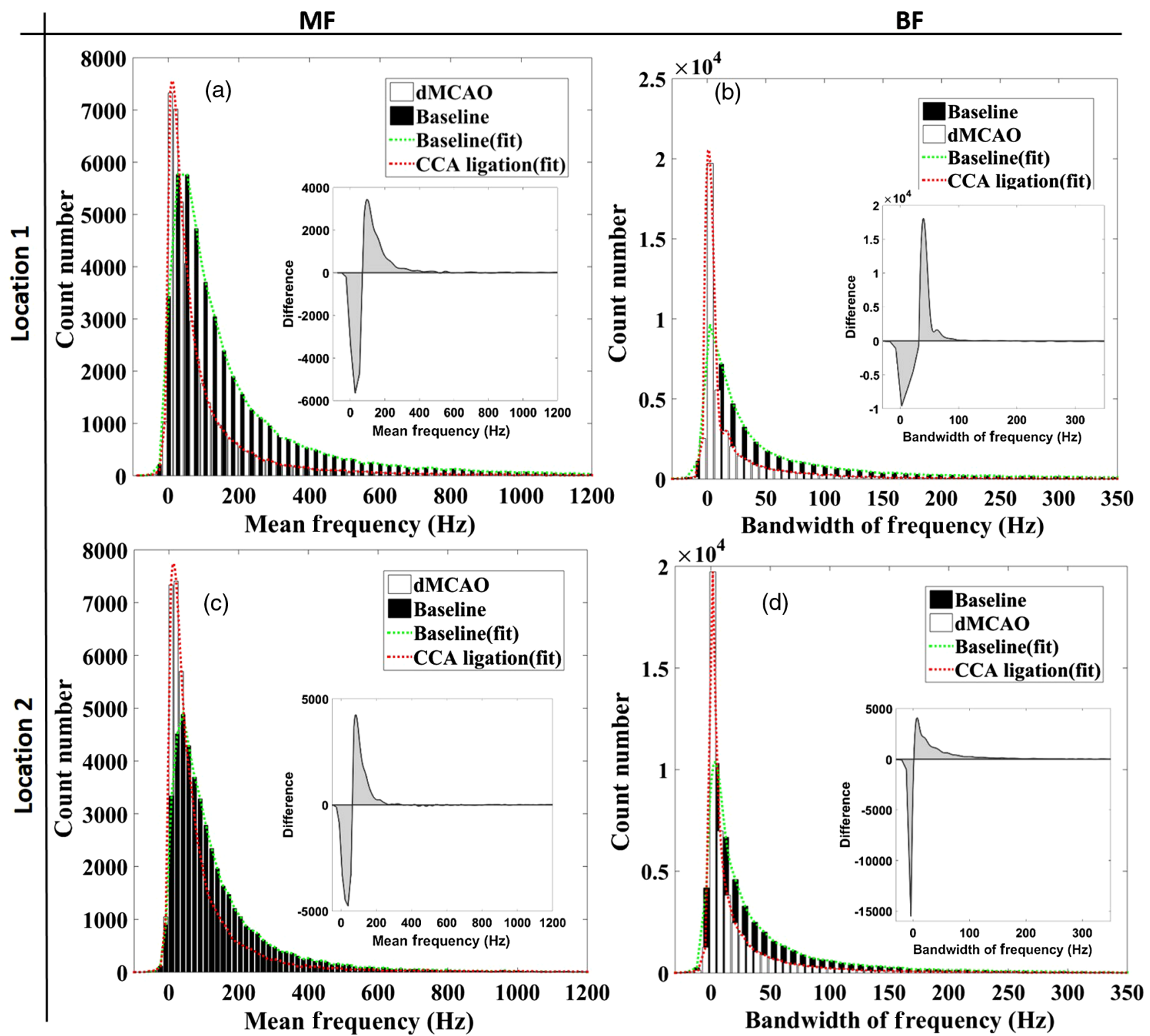

Fig. 8 Statistical analyses for capillary blood flow response to the ischemic injury for both locations 1 and 2. The same ROI is chosen before and after the dMCA ligation (see Fig. 6 for ROls used for quantification). The quantification was performed to provide histogram distribution of the (a, c) MF and (b, d) BF, which indicate the spatial heterogeneity of capillary flows. The BF map indicates directly the temporal heterogeneity of the flow. The insets are the results of differentiation between the histogram functions before and after the dMCA ligation, where the negative value in the curve indicates the increase of the probability within this region, and the positive indicates the opposite. The enface image size is $1.4 \times 1.4 \mathrm{~mm}^{2}$

brain activities while under the ischemic condition. The investigation of this exceeds the scope of the current study. Our purpose here is to demonstrate whether the ED-based quantitative algorithm is useful for providing quantitative information about the cerebral blood flow response to the known tissue injury, which is the dMCAO model in our case.

\section{Discussion}

We have proposed and demonstrated a useful method that is capable of quantifying the hemodynamics of capillary blood flows using the model-based eigencomposition approach with OCTA scanning protocol, which evaluates the eigenvalues and eigenvectors of the ensemble scans (repeated scans at the same location). In mathematics, eigenvalues are a special set of scalars associated with a linear system of equations (i.e., a matrix equation) that are sometimes also known as characteristic roots or characteristic values. An eigenvector corresponds to a real, nonzero eigenvalue point in a direction that is stretched by the transformation while the eigenvalue is the factor by which it is being stretched. In other words, the eigendecomposition analyses of the OCT signals are comprehensive and statistical analyses of the signal features embedded with the ensembles; in this study, the feature of interests is the capillary blood flow.

In the current study, the eigenvalues were calculated from the covariance matrix among the repeated A-lines/B-scans to 
differentiate the static tissue signal and blood flow signal based on the energy of each eigenvalue. The eigenvalue distribution is a measure of the signal spectrum. Each eigenvector and its corresponding eigenvalue represent a part of the signal to be analyzed. At the same time, the corresponding eigenvectors provide the direction for each eigenvalue, i.e., phase information in OCT signal, which is related to the velocity of moving particles. By evaluating the phase changes between eigenvectors, the relative velocity/frequency of dynamic OCT speckle signal due to the blood flow can be estimated. Each eigenvector is related to a specific frequency band. From the eigenvalue/energy spectrum, the static signal originates from stationary and slowly moving tissue or particles, which dominate the low-frequency components and concentrate in a few eigenvalues corresponding to low-frequency eigenvectors. By contrast, the signal from the faster moving blood has most of the energy concentrated along eigenvectors with higher frequency component.

Considering the relationship between dynamic OCT signal and the blood flow velocity, a number of studies ${ }^{38,53}$ reported that, to provide quantifiable information about the capillary flow that is of a wide range from 0 to $5 \mathrm{~mm} / \mathrm{s}$, a relative short time interval is required to capture the dynamic OCT signals due to RBC movements in the capillary vessels. Choi et al. ${ }^{53}$ found a linear relationship between the dynamic OCT signal and flow velocity with a range from 0 to $5 \mathrm{~mm} / \mathrm{s}$ if the OCT system captures the OCT signal at a time interval of $\sim 50 \mu \mathrm{s}$. This critical information dedicates the design of the scanning protocol in this current study. With the time interval of $50 \mu$ s, i.e., the scanning speed of $20 \mathrm{kHz}$ A-scan rate, the results confirmed the findings by Choi et al. (see Fig. 5 from the purposely designed phantom study). This protocol required repeated A-scans at the same position (i.e., M-B mode) rather than the repeated B-scans scanning protocol (i.e., B-M mode) used in the standard OCTA imaging. At the current OCTA system imaging speed of up to $400 \mathrm{kHz}$, the time interval between repeated B-scans is typically around the millisecond range, a value that only provides a possibility of quantifying the flow speed up to $300 \mu \mathrm{m}$ per second (according to Choi et al.), ${ }^{53}$ which would not be suitable for quantifying the capillary blood flow within tissue beds that often cover a range from 0 to $5 \mathrm{~mm} / \mathrm{s}$. Therefore, with the current OCT system designed for OCTA imaging, the M-B scanning protocol perhaps is the only feasible solution to provide quantitative information about the capillary blood flows within tissue beds. However, with the rapid development of swept laser sources that has pushed the OCT imaging speed up to a few MHz A-scan rate, ${ }^{8,9}$ it is hopeful that the standard OCTA scanning protocol would be amendable in near the future to provide quantitative information of capillary blood flows.

We have attempted to use the proposed ED-based quantification algorithm to investigate the capillary blood flow response to an ischemic brain injury. It is the first time that the eigenvectors of an ensemble scan were utilized to estimate the MF and the BF of the dynamic OCT signals due to the moving RBCs in the capillary vessel. The MF and BF are two important parameters that would provide an ability to characterize the capillary blood flow heterogeneity within the cortical tissue in rodents. Although the results are promising and, more importantly, the relationship between MF and velocity is found to be linear, the exact relation between MF and velocity has not yet been explored. Other than the flow speed, other factors may be involved in this relationship, for example, the actual size, shape, and orientation of RBCs, as well as the OCT beam spot size.
The rigorous investigation of this relation is, however, beyond the scope of the current study.

Although we have demonstrated the ED-based quantification algorithm in the estimation of MF and BF of the capillary blood flows within tissue beds, a number of limitations exist. The number of eigenvalues is dependent on the ensemble number of the repeated scans. It is known that the more the repeated scans, the more eigenvalues and eigenvectors there would be and, therefore, the more frequency bands would be available for analysis. According to Fig. 3, the frequency estimation tends to be saturated in the last few of eigenvectors. Considering the spectrum distribution of eigenvalues (Fig. 2) and the frequency distribution associated with the eigenvalues (Fig. 3), only the first 15 eigenvalues actually contribute to the efficient frequency estimations. Further study would be necessary to optimize the number of repeated scans required for the accurate estimation of the MF and BF of the dynamic OCT signals due to the moving RBCs. Another limitation is that the M-B scanning protocol is not compatible with the current commercial OCTA systems that use the standard B-M scanning protocol. This limitation is likely mitigated using an $\mathrm{MHz}$ swept laser source in the OCTA system, which however is a long way from a commercially viable system. Therefore, unless an M-mode scanning protocol is used, it seems that it is almost impossible to utilize the B-M mode scanning protocol available in commercial systems to realize the velocimetry of capillary blood flows.

\section{Conclusion}

We have demonstrated a useful method for the velocity estimation of capillary blood flows with OCTA scans. The method utilized a model-based ED statistical approach that is capable of extracting the dynamic complex OCT signals due to the moving RBCs, upon which the frequencies of eigenvalues that are related to the RBC moving velocity are statistically estimated. We have shown that the ED-based analyses are capable of providing three important parameters about the dynamic flow signal, including the power of the spectrum, the MF, and the bandwidth of the frequency of the blood flow, each corresponding to the OCTA intensity signal, velocity, and the velocity bandwidth of blood flow. We have demonstrated the linear relationship between the estimated MF and the flow velocity using a purposely designed microfluidic phantoms and explored the usefulness of the proposed ED-based algorithm in the quantitative investigation of capillary flow responses to an ischemic stroke model. Further directions for this development include the systematic investigation of the relationship between the MF and the actual flow speed, and the systematic exploration of the utility of the proposed method in neuroscience, ophthalmology, and dermatology, where tissue injury and therapeutic treatment are known to have vascular involvements.

\section{Disclosures}

At the current stage, the authors declare no competing interests in the method discussed in this paper.

\section{Acknowledgments}

This work was supported in part by grants from the National Heart, Lung, and Blood Institute (No. R01HL093140) and the National Eye Institute (No. R01EY024158). The content is solely the responsibility of the authors and does not necessarily represent the official views of grant-giving bodies. 


\section{References}

1. A. Fercher et al., "Optical coherence tomography-principles and applications," Rep. Prog. Phys. 66(2), 239-303 (2003).

2. P. H. Tomlins and R. K. Wang, "Theory, developments and applications of optical coherence tomography," J. Phys. D Appl. Phys. 38(15), 2519 (2005).

3. W. Drexler et al., "Ultrahigh-resolution ophthalmic optical coherence tomography," Nat. Med. 7(4), 502-507 (2001).

4. R. Leitgeb, C. K. Hitzenberger, and A. F. Fercher, "Performance of Fourier domain vs. time domain optical coherence tomography," Opt. Express 11(8), 889-894 (2003).

5. M. A. Choma et al., "Sensitivity advantage of swept source and Fourier domain optical coherence tomography," Opt. Express 11(18), 21832189 (2003).

6. J. F. de Boer et al., "Improved signal-to-noise ratio in spectral-domain compared with time-domain optical coherence tomography," Opt. Lett. 28(21), 2067-2069 (2003).

7. L. An et al., "High speed spectral domain optical coherence tomography for retinal imaging at 500,000 A-lines per second," Biomed. Opt. Express 2(10), 2770-2783 (2011).

8. T. Klein et al., "Megahertz OCT for ultrawide-field retinal imaging with a 1050nm Fourier domain mode-locked laser," Opt. Express 19, 3044-3062 (2011).

9. J. Xu et al., "High-performance multi-megahertz optical coherence tomography based on amplified optical time-stretch," Biomed. Opt. Express 6, 1340-1350 (2015).

10. J. A. Izatt et al., "In vivo bidirectional color Doppler flow imaging of picoliter blood volumes using optical coherence tomography," Opt. Lett. 22(18), 1439-1441 (1997).

11. Z. Chen et al., "Optical Doppler tomographic imaging of fluid flow velocity in highly scattering media," Opt. Lett. 22(1), 64-66 (1997).

12. Y. Wang et al., "In vivo total retinal blood flow measurement by Fourier domain Doppler optical coherence tomography," J. Biomed. Opt. 12(4), 041215 (2007).

13. Z. Zhi et al., "Volumetric and quantitative imaging of retinal blood flow in rats with optical microangiography," Biomed. Opt. Express 2(3), 579-591 (2011).

14. W. J. Choi et al., "Measurement of pulsatile total blood flow in the human and rat retina with ultrahigh speed spectral/Fourier domain OCT," Biomed. Opt. Express 3(5), 1047 (2012).

15. V. J. Srinivasan et al., "Quantitative cerebral blood flow with optical coherence tomography," Opt. Express 18(3), 2477-2494 (2010).

16. H. M. Subhash et al., "Volumetric in vivo imaging of microvascular perfusion within the intact cochlea in mice using ultra-high sensitive optical microangiography," IEEE Trans. Med. Imaging 30, 224-230 (2011).

17. C. Mason et al., "Doppler optical coherence tomography for measuring flow in engineered tissue," Biosens. Bioelectron. 20, 414-423 (2004).

18. S. G. Proskurin, Y. He, and R. K. Wang, "Determination of flow velocity vector based on Doppler shift and spectrum broadening with optical coherence tomography," Opt. Lett. 28(14), 1227-1229 (2003).

19. Y. Zhao et al., "Phase-resolved optical coherence tomography and optical Doppler tomography for imaging blood flow in human skin with fast scanning speed and high velocity sensitivity," Opt. Lett. 25(2), $114-116$ (2000).

20. S. Makita et al., "Optical coherence angiography," Opt. Express 14(17), 7821-7840 (2006).

21. R. K. Wang et al., "Three dimensional optical angiography," Opt. Express 15(7), 4083-4097 (2007).

22. R. K. Wang et al., "Depth-resolved imaging of capillary networks in retina and choroid using ultrahigh sensitive optical microangiography," Opt. Lett. 35(9), 1467-1469 (2010).

23. L. An, J. Qin, and R. K. Wang, "Ultrahigh sensitive optical microangiography for in vivo imaging of microcirculations within human skin tissue beds," Opt. Express 18(8), 8220-8228 (2010).

24. A. Mariampillai et al., "Speckle variance detection of microvasculature using swept-source optical coherence tomography," Opt. Lett. 33(13), 1530-1532 (2008).

25. Y. Jia et al., "Split-spectrum amplitude-decorrelation angiography with optical coherence tomography," Opt. Express 20(4), 4710-4725 (2012).
26. D. Yu Kim et al., "In vivo volumetric imaging of human retinal circulation with phase-variance optical coherence tomography," Biomed. Opt. Express 2(6), 1504-1513 (2011).

27. J. Enfield, E. Jonathan, and M. J. Leahy, "In vivo imaging of the microcirculation of the volar forearm using correlation mapping optical coherence tomography (cmOCT)," Biomed. Opt. Express 2(5), 1184-1193 (2011).

28. A. Zhang et al., "Methods and algorithms for optical coherence tomography based angiography: a review and comparison," J. Biomed. Opt. 20(10), 100901 (2015).

29. C. L. Chen and R. K. Wang, "Optical coherence tomography based angiography [Invited]," Biomed. Opt. Express 8(2), 1056-1082 (2017).

30. Y. P. Huang et al., "Swept-source OCT angiography of the retinal vasculature using intensity differentiation based OMAG algorithms," Ophthalmic Surg. Lasers Imaging Retina 45(5), 382-389 (2014).

31. M. R. Thorell et al., "Swept-source OCT angiography of macular telangiectasia type 2," Ophthalmic Surg. Lasers Imaging Retina 45(5), 369-380 (2014).

32. Q. Zhang et al., "Swept source OCT angiography of neovascular macular telangiectasia type 2," Retina 35(11), 2285-2299 (2015).

33. Q. Zhang et al., "Wide-field imaging of retinal vasculature using optical coherence tomography-based microangiography provided by motion tracking," J. Biomed. Opt. 20(6), 066008 (2015).

34. Q. Zhang et al., "Wide-field optical coherence tomography based microangiography for retinal imaging." Sci. Rep. 6, 22017 (2016).

35. D. Obrist et al., "Red blood cell distribution in simplified capillary networks," Philos. Trans. R. Soc. A 368, 2897-2918 (2010).

36. V. J. Srinivasan et al., "OCT methods for capillary velocimetry," Biomed. Opt. Express 3(3), 612-629 (2012).

37. Y. Wang and R. K. Wang, "Autocorrelation optical coherence tomography for mapping transverse particle-flow velocity," Opt. Lett. 35, 3538-3540 (2010).

38. W. J. Choi et al., "Cerebral capillary velocimetry based on temporal OCT speckle contrast," Biomed. Opt. Express 7(12), 4859-4873 (2016).

39. J. Lee et al., "Multiple-capillary measurement of RBC speed, flux, and density with optical coherence tomography," J. Cereb. Blood Flow Metab. 33(11), 1707-1710 (2013).

40. M. Unekawa et al., "Frequency distribution function of red blood cell velocities in single capillaries of the rat cerebral cortex using intravital laser-scanning confocal microscopy with highspeed camera," Asian Biomed. 2(3), 203-218 (2008).

41. M. H. Hayes, Statistical Digital Signal Processing and Modeling, John Wiley \& Sons, Inc., New York (1996).

42. S. Yousefi and R. K. Wang, "Simultaneous estimation of bidirectional particle flow and relative flux using MUSIC-OCT: phantom studies," Phys. Med. Biol. 59(22), 6693-6708 (2014).

43. S. Yousefi, J. Qin, and R. K. Wang, "Super-resolution spectral estimation of optical micro-angiography for quantifying blood flow within microcirculatory tissue beds in vivo," Biomed. Opt. Express 4(7), 1214 (2013)

44. H. Torp, K. Kristoffersen, and B. Angelsen, "Autocorrelation technique in color flow imaging, signal model and statistical properties of the autocorrelation estimates," IEEE Trans. Ultrason. Eng. 41, 604-612 (1994).

45. J. D. Brier, "Laser Doppler and time-varying speckle: a reconciliation," J. Opt. Soc. Am. A. 13(2), 345-350 (1996).

46. R. K. Wang, "Modelling optical properties of soft tissue by fractal distribution of scatterers," J. Mod. Opt. 47(1), 103-120 (2000).

47. R. K. Wang, "Signal degradation by multiple scattering in optical coherence tomography of dense tissue: a Monte Carlo study towards optical clearing of biotissues," Phys. Med. Biol. 47(13), 2281-2299 (2002).

48. T. K. Moon and W. C. Stirling, Mathematical Methods and Algorithms for Signal Processing, Prentice Hall, Upper Saddle River, New Jersey (2000).

49. A. Papoulis, Probability, Random Variables, and Stochastic Processes, 3rd ed., McGraw-Hill, New York (1991).

50. S. Yousefi, Z. Zhi, and R. K. Wang, "Eigendecomposition-based clutter filtering technique for optical microangiography," IEEE Trans. Biomed. Eng. 58(8), 2316-2323 (2011).

51. C. Kasai et al., "Real-time two dimensional blood flow imaging using an autocorrelation technique," IEEE Trans. Sonics Ultrason. 32(3), 458-464 (1985). 
52. Y. Benjamini, “Opening the box of a boxplot," Am. Stat. 42(4), 257-262 (1988).

53. W. J. Choi et al., "Characterizing relationship between optical microangiography signals and capillary flow using microfluidic channels," Biomed. Opt. Express 7(7), 2709-2728 (2016).

54. A. Rosell et al., "Distal occlusion of the middle cerebral artery in mice: are we ready to assess long-term functional outcome?" Transl. Stroke Res. 4(3), 297-307 (2013).

55. Y. Li, U. Baran, and R. K. Wang, "Application of thinned-skull cranial window to mouse cerebral blood flow imaging using optical microangiography," PLoS One 9(11), e113658 (2014).
56. U. Baran, Y. Li, and R. K. Wang, "Vasodynamics of pial and penetrating arterioles in relation to arteriolo-arteriolar anastomosis after focal stroke," Neurophotonics 2(2), 025006 (2015).

57. R. Reif et al., "Quantifying optical microangiography images obtained from a spectral domain optical coherence tomography system," Int. J. Biomed. Imaging 2012, 1-11 (2012).

58. Z. D. Chu et al., "Quantitative assessment of the retinal microvasculature using OCT angiography," J. Biomed. Opt. 21(6), 066008 (2016).

Biographies for the authors are not available. 